\title{
Microscopic Study of Lectin-Crosslinked Amylopectin Gels
}

\author{
Ellina Kesselman, Asher Shazman, Katja Mnich, Eyal Shimoni. \\ Department of Biotechnology and Food Engineering, Technion - Israel Institute of Technology, \\ Haifa, 32000, Israel.
}

The ability of starch molecules to associate in an aqueous medium and create three-dimensional networks, i.e. gelation of starch, is an essential property used in food, pharmaceutical and agricultural applications. Starch gels can be formed by physical interactions between starch polysaccharide chains, or by covalent cross linking [1]. In the current project we study a new concept of forming starch gels: by a lectin-based specific cross linking. Microstructure of these gels determines their physical and mechanical properties, enables the development of a controlled drug delivery and other important applications. This work is aimed to characterize the microstructure of lectin cross linked starch gels using several microscopy techniques.

The gels were formed in a three-component system: amylopectin (of Waxy starch), concanavalin A (a lectin from jack beans) and ions $\mathrm{Mg}^{2+}$ and $\mathrm{Ca}^{2+}$ as salts. Light microscopy (LM), Atomic Force Microscopy (AFM), Scanning Electron Microscopy (SEM) and High-Resolution CryoSEM (HR-Cryo-SEM) were used to characterize the gel microstructure.

The results demonstrated that amylopectin / Concanavalin A gels had very smooth topography and were much more homogeneous than any of their components. This suggests a fine ordering at a molecular level (Figure 1). Nanostructure of the gels was imaged at a lateral resolution less than $5 \mathrm{~nm}$, and a height resolution less than $1 \mathrm{~nm}$ using AFM. Amylopectin-consisting samples showed small nanometric-size protrusions on their surface, which looked like organized biopolymer molecules. These features could be proteins, originated from starch biosynthesis enzymes, remained in the amylopectin, and phase-separated to the surface during gelation process [2]. Although SEM of dried gels showed high porosity at the range of tens of micrometers, HR-Cryo-SEM revealed smooth gel surface, no porosity, and in some places fiberlike pattern, revealed better when irradiated at a high magnification (Figure 2).

The results showed that amylopectin gels, cross linked by Concanavalin A, possessed a continuous and homogeneous structure even at the nanometric scale, which indicate the creation of the gel, highly ordered at the molecular level.

\section{References}

[1] J. N. BeMiller, R. L. Whistler, Starch: Chemistry and Technology, 4rd ed., Food Science and Technology, International Series, Elsevier, 2009.

[2] A. Rindlav-Westling and P. Gatenholm, Biomacromolecules, 4 (2003) 166. 

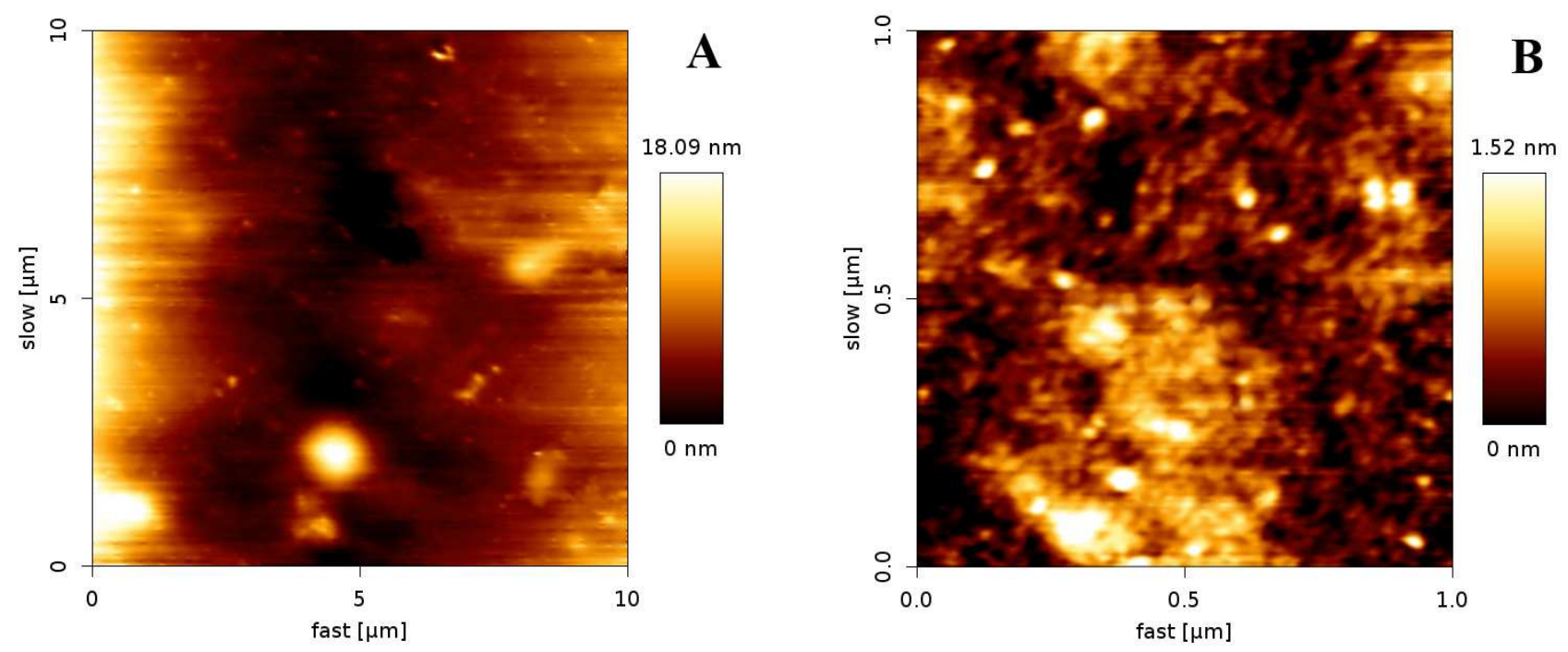

FIG. 1. AFM of dried $8 \%$ amylopectin gel, crosslinked with Con A, imaged in the intermittent contact mode in air. $10 \times 10-\mu \mathrm{m}$ scan (A) and $1 \times 1-\mu \mathrm{m}$ scan (B) show smooth and very low, nmheight topography. At 1- $\mu \mathrm{m}$ scan area the protrusions $\sim 20$-nm width and a few angstroms of height are seen all over the surface. They likely are proteins, originated from starch biosynthesis enzymes.

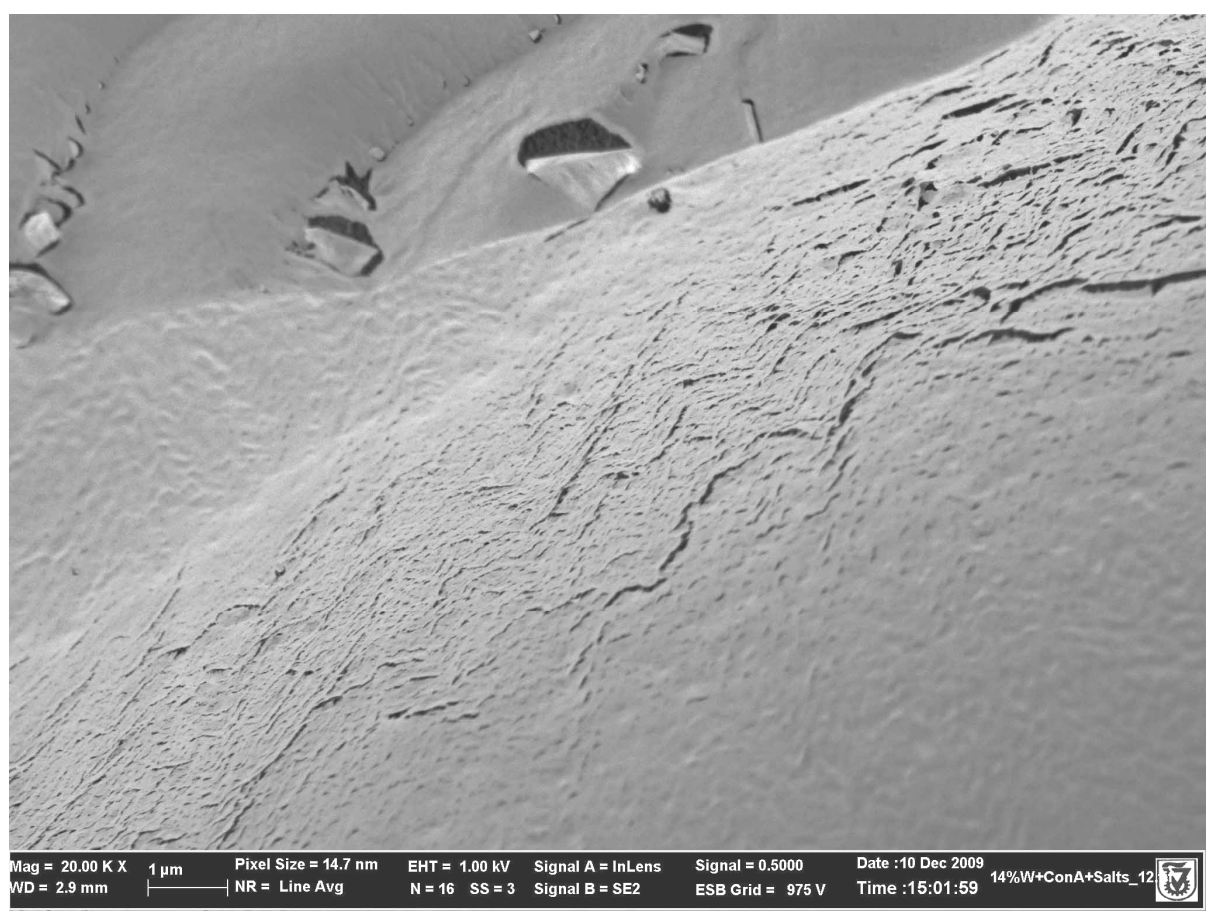

FIG. 2. HR-Cryo-SEM of vitrified and fractured 14\% amylopectin gel, cross linked with Concanavalin A, at the magnification $20000 \times$. Left upper and right lower corners show flat and smooth surface. At the slope (center) a fiber-like pattern is seen. 